\title{
Rapid Nanometer Mapping of Nickel-Steel Friction Stir Weld Joint
}

Genevieve Lee ${ }^{1,2}$, Guilherme Abreu-Faria ${ }^{1,3}$, Johnnatan Rodríguez F. ${ }^{4}$, Jonathan Orsborn ${ }^{5,6}$, Antonio J. Ramirez $^{1,3}$

${ }^{1}$ Welding Eng. Program, Dept. of Materials Science and Eng., The Ohio State University - Columbus, $O H, U S A$

${ }^{2}$ Center for Design and Manufacturing Excellence, College of Engineering, The Ohio State UniversityColumbus, $\mathrm{OH}$, USA.

${ }^{3}$ Manufacturing and Materials Joining Innovation Center - Ma ${ }^{2}$ JIC, Dept. of Materials Science and Eng., The Ohio State University - Columbus, OH, USA

${ }^{4}$ Dept. of Mechanical Engineering, EIA University - Envigado, Ant., Colombia

${ }^{5}$ Center for Electron Microscopy, Dept. of Materials Science and Eng., The Ohio State University Columbus, $\mathrm{OH}, \mathrm{USA}$

${ }^{6}$ Center for the Accelerated Maturation of Materials, Dept. of Materials Science and Eng., The Ohio State University, Columbus, $\mathrm{OH}$

Detailed nanometer-scale characterization of large bulk materials can prove to be an expensive and tedious task on the transmission electron microscope (TEM), even for skilled microscopists using state of the art systems. For example, when dissimilar material joints need to be evaluated at the micro- and nanoscale to verify features at the interface that may be deleterious, the analysis of one single TEM sample may not provide the right statistically relevant information needed to understand the expected joint performance. Friction stir welded joints have been reported to present sub-micron microstructural features originated by the intense shearing and plasticization that the rotating tool imparts to the processed material during such solid-state joining processes. This necessitates high-resolution techniques for appropriate, thorough characterization and understanding of the evolution and performance of such joints. Transmission Kikuchi diffraction (TKD), also referred to as transmission electron backscatter diffraction (t-EBSD), is a scanning electron microscopy (SEM) technique that can perform materials analysis down to $10 \mathrm{~nm}$ resolution in quite efficiently [1]. TKD, similar to EBSD, provides crystallographic data, and it can be easily paired with energy dispersive X-ray spectroscopy (XEDS) for more comprehensive characterization. Such analyses provide maps containing grain, grain boundary, crystal orientation, strain, phase, and chemical information that can provide better understanding of the sample before conducting more localized studies including TEM which provide detailed information down to the atomic scale, but a limited understanding of the entire sample. Therefore, TKD provides a better holistic understanding of the sample area and can significantly reduce the time and effort required in the TEM once a thin foil has been created.

In the oil and gas industry, high quality materials and weldments are necessary for sub-sea structures as failure can result in severe environmental and economic consequences. Friction stir welding offers joints of superior mechanical performance compared to conventional fusion welding. However for such essential joints, quality must be ensured throughout the entirety of the joint. Dissimilar metal FSW microstructures between nickel-based alloys and carbon steels have not been extensively reported. This study delves into the interface morphology and localized textures that are present in the stir zone of a FSW between Ni-based alloy 625 and plain carbon steel A516.

A composite W-Re matrix reinforced with cubic boron nitride (PCBN) pin tooling was used to friction stir weld Ni-based alloy 625 and A516 carbon steel plates [2]. Characterization was conducted using 
optical and electron microscopy imaging on the joint bulk cross section. A thin foil was extracted and thinned at the local joint interface using a focused-ion beam (FIB) and polished with low-energy ion milling. Morphological, crystallographic and chemical information across the interface was gathered using TKD and XEDS and complemented with TEM analysis of nanometer-size precipitates. Simultaneous TKD and XEDS information collected is shown in Fig. 2. Reduction in both steel and Nibased alloy grain size is observed immediately adjacent to the interface. At the steel side of the joint, a layer of fine $(\sim 300 \mathrm{~nm})$ and highly deformed ferrite grains is present. Conversely, large austenite grains $(\sim 1 \mu \mathrm{m})$ with $\mathrm{Nb}$ - and Mo-rich precipitates $(50-150 \mu \mathrm{m})$ were observed at the Ni-based alloy side. The $\mathrm{Nb}$-rich precipitates were identified by TKD and XEDS as NbC carbides. The Mo-rich precipitates were identified as $\mathrm{Mo}_{6} \mathrm{C}$ carbides using TEM. Additionally, localized texture within micrometers adjacent to the FSW interface was characterized, as seen in Fig. 3. [3].

TKD of a dissimilar FSW joint between Ni-based alloy 625 and plain carbon steel easily provides a picture of the interface that is unattainable with only EBSD or TEM analysis. Grain size, grain and precipitate orientations, precipitate type and more can be efficiently and timely identified on the same thin foil subsequently used for TEM characterization of specific areas previously analyzed on the SEM.

\section{References:}

[1] Keller, R R, and R H Geiss. Journal of Microscopy 245, no. 3 (2012): 245-251.

[2] J.R. Rodriguez and A.J. Ramirez, Materials Characterization 110 (2015), p.126-135.

[3] R. W. Fonda and K. E. Knipling, Science and Technology of Welding and Joining, p. 288, 2011.

[4] The authors acknowledge funding from the Center for Design and Manufacturing Excellence

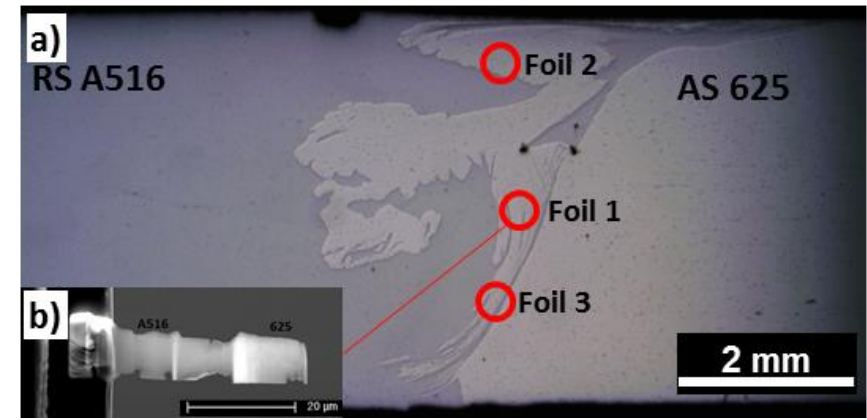

Figure 1. Dissimilar friction stir weld a) cross section taken with optical microscopy and b) extracted FIB foil taken with SEM imaging at the interface of Ni-base alloy 625 and carbon steel A516

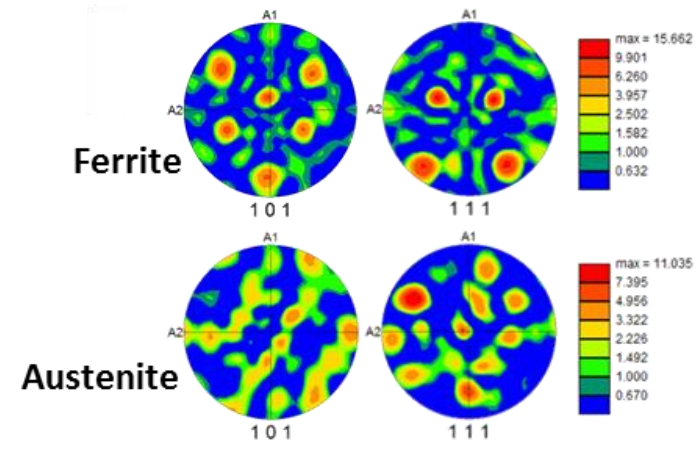

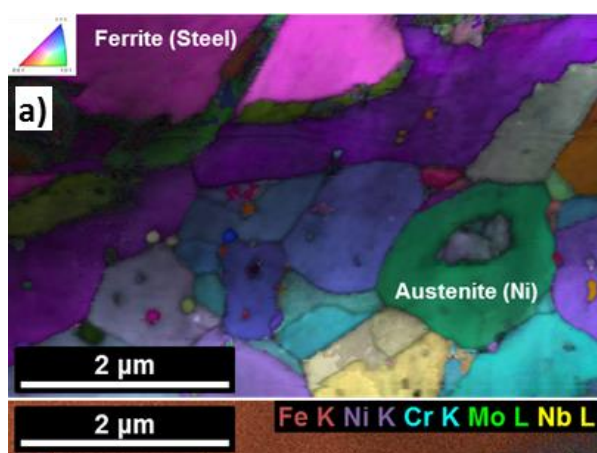
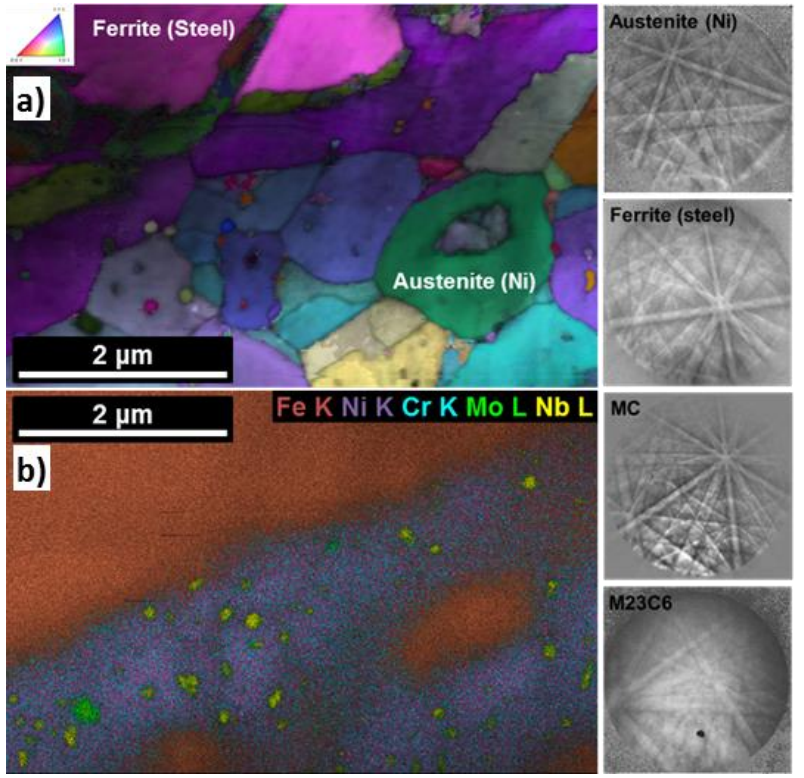

Figure 2. a) IPF (N) maps taken using TKD in SEM. Diffraction patterns of 4 separate phases identified are listed at the right. b) EDS maps of the same scanned area shows compositional information of phases and precipitates. Mo-and Nb-rich precipitates can be seen.

Figure 3. Pole figure for ferrite and austenite phase of the scan in Figure 2. Pole figures at 101 and 111 are shown for both. Maximum texture is approximately 15.6 and 11.0 times random in the ferrite and austenite, respectively. 